\title{
Numerical Characteristics of Vehicle Collision Speed and Acceleration Peak
}

\author{
Ying Lu*, Zhenyu Chen and Zhuang Zhou \\ School of Automotive and Traffic Engineering, Jiangsu University, Zhenjiang, China \\ ${ }^{*}$ Corresponding author
}

\begin{abstract}
In order to improve road safety, the probability distribution of vehicle collision speeds and acceleration peaks were investigated in this paper. First, based on statistical data from the National Highway Traffic Safety Administration in the U.S., the probability density function of vehicle collision speeds was worked out. Next, a finite element model of trolley impact system was built. Through analyzing data generated by this simulation model, a regression model was developed to reveal the association between collision speed and acceleration peak. Then, the expectation and variance of acceleration peak were calculated. The results show that collision speeds follow a normal distribution and there is the biggest possibility of collision accidents happening at middle and low speeds. The numerical characteristics of acceleration peak are useful for optimizing of automobile safety system under the condition of random impact.
\end{abstract}

Keywords—vehicle collision; speed analysis; acceleration peak

\section{INTRODUCTION}

As the number of cars increases in cities, there are more and more traffic accidents on the road. The report from World Health Organization (WHO) finds that around 1.25 million people die every year as a result of road traffic crashes. Speedrelated road traffic crashes remain the number one cause of death among people. Although typically $40-50 \%$ of drivers go over posted speed limits in traffic accident, the U.S. Department of Transportation's Federal Highway Administration has concluded that the risk of having a crash is increased both for vehicles traveling slower than the average speed, and for those traveling above the average speed. On the other hand, acceleration peak, which is high correlated with the collision speed, is significant for designing active and passive automotive safety systems. Due to the randomness of collision speed, the value of acceleration peak has to be examined based on statistical analysis. It is also useful to investigate the distribution and characteristics of collision speed and acceleration peak for optimizing vehicle design and improving the survival rate of passengers in collision accidents.

Numerous studies have examined the relationship between vehicle speed and the severity of traffic crashes. One major research interest focuses on speed limits through statistical approaches. Fagerlind et al.[1] investigated road crashes sampled in a Swedish region. Crash related variables in their research included 'Crash type', 'Posted speed limit', and 'Number of vehicles in crash'. Huang et al.[2] analyzed the historical crash dataset in Florida in 2007 and developed a Bayesian bivariate hierarchical ordered logistic model to estimate the occupant protectiveness and vehicle protectiveness where car brands and speed limit were both important control variables. Zou and Tarko[3] developed a crash modification factors and estimated the average crash costs applicable to a wide range of road-barrier scenarios. Hamzeie et al.[4] analyzed speed data to determine the effects of speed limit policies on the mean and standard deviation of travel speeds. Zeng et al.[5] used crash data set from the Florida to investigate the interaction of driver vehicle units and analyzed the twovehicle crashes where speed limit was an important factor.

Considering speed is one of the main crash contributory factors of road accidents, another major research interest is the evaluation of occupant injure in accident. Bener et al.[6] determined the effects of aggressive behaviors (such as careless driving, excessive speed, driver sleepiness and fatigue) on injuries among Turkish population. Imprialou et al.[7] developed a model of injury severity using Poisson lognormal regression, which involved vehicle speeds. The purpose of their research was to make lawmakers consider increasing speed limit.

There are also a lot of papers that directly focus on the characteristics of collision speed by applying statistical methods. Vadeby and Forsman[8] recorded speeds of more than 300000 vehicles and present power and exponential models of vehicle speeds. $\mathrm{Hu}[9]$ developed a Log-linear regression model to estimate the effect of increased speed limit on vehicle speed and speed variance. They found increasing speed limit lead to higher travel speed and an increased probability of exceeding the new speed limit.

From the literature mentioned above, we can see that much research aims to improve the scientificalness of government decision-making on laws and regulations of road traffic instead of safety equipment in vehicles. To some extent, our research is quite similar to those focusing on the distribution of collision speeds. However, the acceleration peak plays a more important role in our research. To the best of our knowledge, there is little research simultaneously analyzing the distribution of vehicle collision speed and acceleration peak, which is vital to determine the technical parameters of some passive automotive safety systems.

The purpose of this study is to present the probability distributions of the vehicle collision speed and the acceleration peak as well as to calculate their expectations and variances, which can be used to optimize vehicle safety systems under random collision conditions. The rest of this paper is organized 
as follows. In Section II we analyze the distribution of speeds in vehicle collision accidents. Section III exams the relationship between collision speed and acceleration peak. Section IV discusses the expectation and variance of the acceleration peak. Finally, Section V presents summary comments and discusses the areas for future research.

\section{ANALYSIS OF DistribUtion OF VeHICLE SPEEDS IN COLLISION ACCIDENTS}

A total of 862 collision records in American cities during thirteen years from 2004 to 2016, which are maintained by the National Highway Traffic Safety Administration (NHTSA) in U.S., are used for the investigation. Typically, we categorize the collision speeds in twenty groups: $0-10,11-20,21-30,31-$ 40, 41-50, 51-60, 61-70, 71-80, 81-90, 91-100, 101-110, 111-120, 121-130, 131-140, 141-150, 151-160, 161-170, 171-180, 181-190 and 191+. The corresponding numbers of collisions for these groups are shown in Table I.

TABLE I. VEHICLE CRASH IN GROUPS

\begin{tabular}{|c|c|c|c|c|c|c|}
\hline $\begin{array}{c}\begin{array}{c}\text { Speed group } \\
(\mathrm{km} / \mathrm{h})\end{array} \\
\end{array}$ & $0-10$ & $11-20$ & $21-30$ & $31-40$ & $41-50$ & $51-60$ \\
\hline $\begin{array}{c}\text { Number of } \\
\text { collisions }\end{array}$ & 45 & 82 & 172 & 117 & 121 & 93 \\
\hline $\begin{array}{c}\text { Speed group } \\
(\mathrm{km} / \mathrm{h})\end{array}$ & $61-70$ & 71-80 & 81-90 & 91-100 & $101-110$ & $111-120$ \\
\hline $\begin{array}{c}\text { Number of } \\
\text { collisions }\end{array}$ & 50 & 58 & 31 & 29 & 20 & 12 \\
\hline $\begin{array}{c}\text { Speed group } \\
(\mathrm{km} / \mathrm{h})\end{array}$ & $121-130$ & $131-140$ & 141-150 & $151-160$ & $161-170$ & $171-180$ \\
\hline $\begin{array}{c}\text { Number of } \\
\text { collisions }\end{array}$ & 10 & 7 & 10 & 1 & 1 & 3 \\
\hline $\begin{array}{c}\text { Speed group } \\
(\mathrm{km} / \mathrm{h})\end{array}$ & 181-190 & $190+$ & & & & \\
\hline $\begin{array}{c}\text { Number of } \\
\text { collisions }\end{array}$ & 1 & 1 & & & & \\
\hline
\end{tabular}

From Table I, it can be seen that in the groups of 21$30 \mathrm{~km} / \mathrm{h}, 31-40 \mathrm{~km} / \mathrm{h}, 41-50 \mathrm{~km} / \mathrm{h}$ there are more than 100 collision accidents, which means the interval between 21 and 50 has the highest accidence rate. However, after the vehicle speed is beyond $150 \mathrm{~km} / \mathrm{h}$, the number of accidents is relatively small, whereas the severity increases.

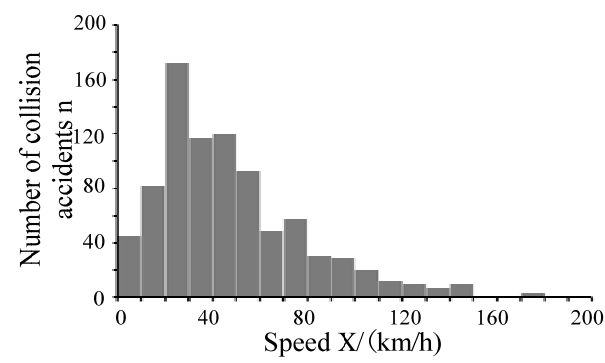

FIGURE I. HISTOGRAM OF COLLISION SPEED

To investigate the distribution of collision speed, we draw a histogram according to Table I, as shown in Figure I. Judging from this histogram, the crash speed is generally consistent with a normal distribution. Trough SPSS software, the vehicle speeds are investigated whether they follow the normal distribution. The results are shown in Table II.

TABLE II. NORMAL DISTRIBUTION VERIFICATION

\begin{tabular}{|c|c|c|}
\hline \multirow{2}{*}{ Fit Index } & \multicolumn{2}{|c|}{ Value } \\
\cline { 2 - 3 } Mean & Statistics & Standard error \\
\hline $\begin{array}{c}\text { 95\% confidence interval } \\
\text { for mean-lower bound }\end{array}$ & 48.48 & 1.08 \\
\hline $\begin{array}{c}\text { 95\% confidence interval } \\
\text { for mean- upper bound }\end{array}$ & 50.60 & - \\
\hline 5\% trimmed mean & 45.82 & - \\
\hline Median & 40.23 & - \\
\hline Variance & 1009.97 & - \\
\hline Standard deviation & 31.78 & - \\
\hline Min & 4.82 & - \\
\hline Max & 196.33 & - \\
\hline Range & 191.51 & 0.08 \\
\hline Interquartile range & 37.01 & -16 \\
\hline Skewness & 1.32 & -96 \\
\hline Peakedness & 1.96 & - \\
\hline & & - \\
\hline
\end{tabular}

Since the skewness is 1.32 and the kurtosis is 1.96 , both of which are less than 2, it can be determined that the collision speed distribution approximately follows a normal distribution. The normal distribution curve has been drawn in the histogram, as shown in Figure II.

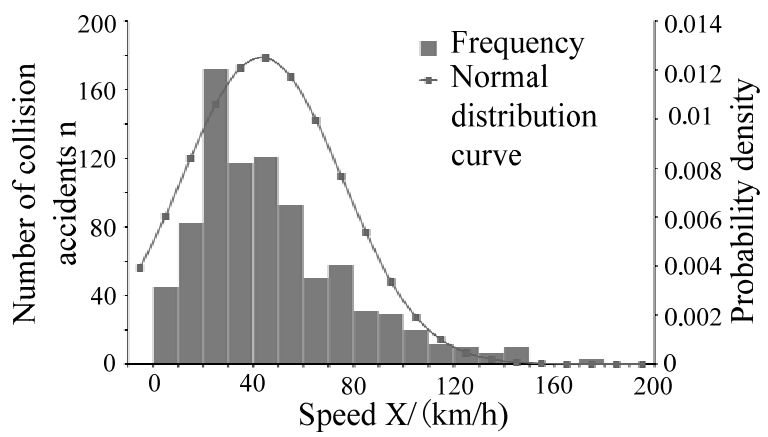

FIGURE II. HISTOGRAM AND NORMAL DISTRIBUTION CURVE OF COLLISION SPEED

From the normal distribution curve, we can see that the collision speed group with maximum probability is $41-50 \mathrm{~km} / \mathrm{h}$. In general, due to few cars running at a high speed, the collision probabilities in the high speed groups are relatively low, while the number of vehicle running at medium and low speeds is large, which leads to a higher crash probability.

From Table II, the mean and standard deviation of crash speeds are 48.48 and 31.78 , respectively. The probability density function of crash speeds is given by 


$$
f(X)=\frac{1}{\sqrt{2 \pi} \times 31.78} e^{-\frac{(X-48.48)^{2}}{2 \times 31.78^{2}}}
$$

where $X$ is collision speed.

Note that there are different standards of estimation of safety speeds in vehicle collision test in different countries. For instance, the frontal collision test speed is set to $48.3 \mathrm{~km} / \mathrm{h}$ in American vehicle safety regulations as well as $50 \mathrm{~km} / \mathrm{h}$ in both Japan's and China's. In this paper, through the study of vehicle collision speed distribution, we figure out that the speed of maximum crash probability is $48.48 \mathrm{~km} / \mathrm{h}$, which is in accordance with current vehicle safety assessment standard in several countries. Hence, the probability distribution of collision speed presented in this paper can provide more accurate data to optimize the vehicle safety system.

\section{THE RELATIONSHIP BETWEEN COLLISION SPEED AND ACCELERATION PEAK}

\section{A. Establishment of Finite Element Simulation Model}

In some cases, the acceleration peak is more important in the design of safety system, especially in those involving acceleration-related algorithm. However, due to the lack of collision accident data, the probability distribution of acceleration peak is difficult to find. Generally speaking, in order to obtain a reasonable relationship between acceleration peak and collision speed, a large number of real-vehicle collision tests at different speeds should be implemented. However, considering the costs of real-vehicle collision tests, we carry out several crash tests through trolley impact system rather than real-vehicles.

DAPG-QJ-MNPZ trolley impact system that we use here is made of No.45 steel. It weighs $400 \mathrm{~kg}, 1800 \mathrm{~mm}$ long, $20 \mathrm{~mm}$ wide and $100 \mathrm{~mm}$ high. The trolley consists of an impact system, an olive heads and some struts. The olive head is 46.4 $\mathrm{mm}$ in diameter, the strut is $27.0 \mathrm{~mm}$ in diameter and 645.0 $\mathrm{mm}$ long. One end of the strut joins the trolley and the other end connects the olive head. During the clash process, the olive heads hit guiding tubes in which there are energy absorbing tubes. The energy absorbing tubes whose outer diameters are $59.1 \mathrm{~mm}$ are made of polyurethane composites. The pore structure of energy absorbing tube is shaped like a truncated cone. The internal diameter of the end of energy absorbing tube that directly bears the impact force is $44.5 \mathrm{~mm}$ and the counterpart of the other is $21.8 \mathrm{~mm}$. In the trolley impact test, two energy absorbing tubes are installed in the guide tube with a length of $655.0 \mathrm{~mm}$, an outer diameter of $80.0 \mathrm{~mm}$ and an inner diameter $59.4 \mathrm{~mm}$. The rigid barrier is $1800 \mathrm{~mm}$ long, 20 $\mathrm{mm}$ wide and $1000 \mathrm{~mm}$ high, which connects with the guide tube, as shown in Figure III.

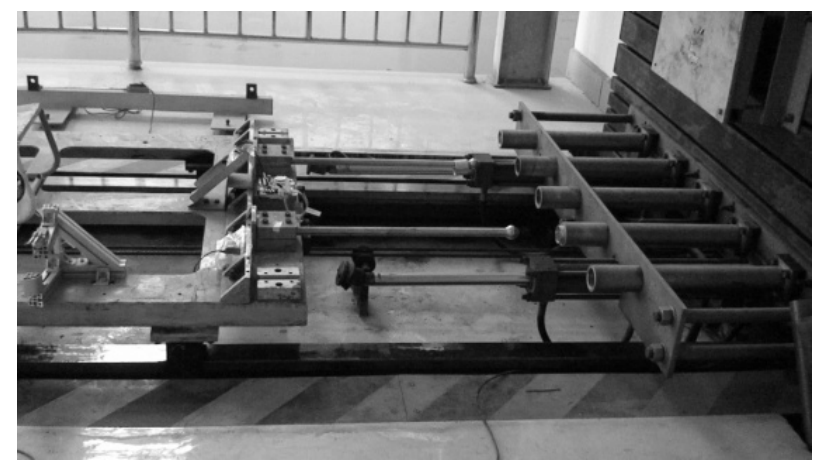

FIGURE III. TROLLEY IMPACT SYSTEM

To generate plenty of data, a finite element simulation model in accordance with DAPG-QJ-MNPZ trolley impact system is built by software LS-DYNA, as shown in Figure IV. We have proved the reliability of this simulation model by comparing the accelerate curves obtained in trolley impact system and those generates in simulation model [10]. This simulation model can repeat the process of trolley impact and calculate the acceleration peak at different collision speeds.

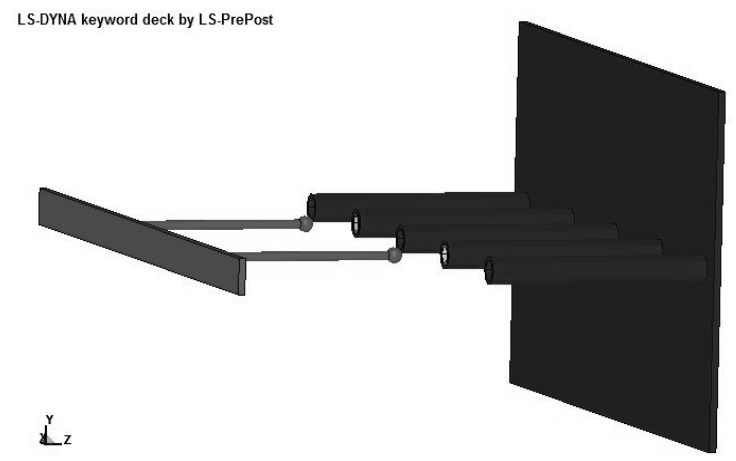

FIGURE IV. FINITE ELEMENT SIMULATION MODEL OF TROLLEY IMPACT SYSTEM

\section{B. The Regression Model about Collision Speed and Acceleration Peak}

The impact tests at different initial speeds are carried out by using simulation model, and the acceleration peaks at different initial speeds are calculated, as shown in Table III.

TABLE III. ACCELERATION PEAK AT DIFFERENT INITIAL SPEEDS

\begin{tabular}{|c|c|c|c|c|c|c|c|c|c|c|}
\hline $\begin{array}{c}\text { Initial } \\
\text { speed } \\
\boldsymbol{X} /(\mathbf{k m} / \mathbf{h})\end{array}$ & 10 & 15 & 20 & 25 & 30 & 35 & 40 & 45 & 50 & 55 \\
\hline $\begin{array}{c}\text { Acceler } \\
\text { ation } \\
\text { peak Y/g }\end{array}$ & 9.1 & 11.0 & 12.6 & 13.5 & 14.3 & 18.6 & 22.5 & 26.8 & 43.2 & 59.5 \\
\hline
\end{tabular}

By analyzing the data in Table III, the relationship between collision speed $X$ and acceleration peak $Y$ are fitted by polynomial model, exponential model and power model, respectively. The functional forms and the coefficients of determination of these models $R^{2}$ are shown in Table IV. The trend lines of these models are shown in Figure V. 
TABLE IV. THE REGRESSION MODEL

\begin{tabular}{|c|c|c|}
\hline $\begin{array}{c}\text { Functional } \\
\text { form }\end{array}$ & Expression & $R^{2}$ \\
\hline polynomial & $Y=0.0365 X^{2}-1.4265 X+23.369$ & 0.9599 \\
\hline exponential & $Y=5.4501 e^{0.0388 X}$ & 0.9343 \\
\hline Power & $Y=0.7099 X^{0.9824}$ & 0.8019 \\
\hline
\end{tabular}

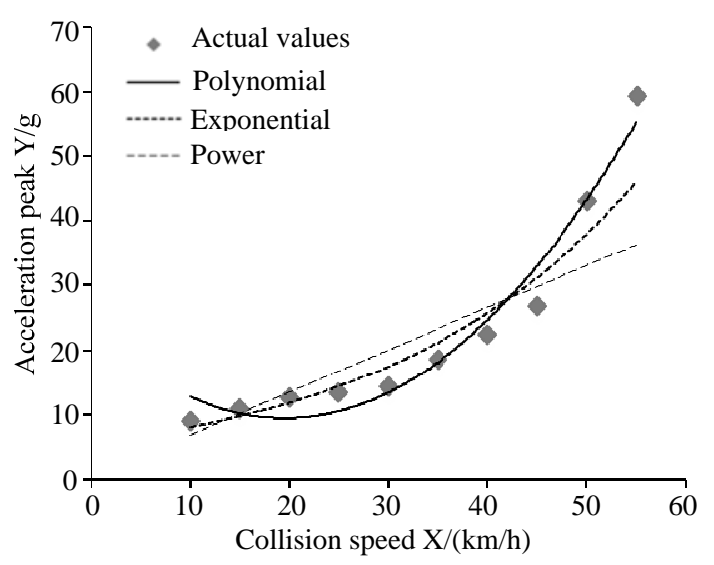

FIGURE V. TREND LINES OF THE MODELS

From Figure V, we can see that the polynomial model has the best fitting effect (the coefficient of determination $R^{2}$ is the largest). Consequently, the polynomial model can be used for further analysis. The expression for acceleration peak $Y$ in terms of the collision speed $X$ is given by

$$
Y=0.0365 X^{2}-1.4265 X+23.369
$$

\section{EXPECTATION AND VARIANCE OF ACCELERATION PEAK}

Naturally, once we have obtained the probability density function of collision speeds and the regression model for acceleration peak $Y$ in terms of collision speed $X$, the probability density function of acceleration peak could be easily worked out. However, the expression of the probability density function of $Y$ tends to be very complex. Considering the expectation and variance of probability distribution play a more important role in optimal design, we calculate both of them by using (1) and (2).

\section{A. Calculation of the Expectation of Acceleration Peak}

Note that the expectation of $X$ is known and acceleration peak $Y=a X^{2}+b X+c$. The expectation of $Y$ can be calculated by using (3).

$$
\begin{aligned}
E(Y) & =E\left(a X^{2}+b X+c\right) \\
& =a E\left(X^{2}\right)+b E(X)+c
\end{aligned}
$$

According to (2), we have $a=0.0365$, $b=-1.4262, c=23.369$. Then substitute these values into (3) to obtain

$$
E(Y)=0.0365 E\left(X^{2}\right)-1.4262 E(X)+23.369
$$

where $E\left(X^{2}\right)$ can be calculated as follows:

$$
E\left(X^{2}\right)=D(X)+[E(X)]^{2}
$$

According (1), $E(X)=48.48, D(X)=1009.9$. Inserting these values into (5), we have $E\left(X^{2}\right)=3360.3$.

Finally, insert the values of $E\left(X^{2}\right)$ and $E(X)$ into (4) to obtain the expectation of the acceleration peak $E(Y)=76.86$.

\section{B. Calculation of Variance of Acceleration Peak}

Since the expectation of acceleration peak $Y$ is known as well as $X$ and $Y$ are not linearly related, the variance of $Y$ can be calculated by using (6):

$$
\begin{aligned}
D(Y) & =D\left(a X^{2}+b X+c\right) \\
& =a^{2} D\left(X^{2}\right)+b^{2} D(X)
\end{aligned}
$$

where $a, b, c$ are determined in the above.$D\left(X^{2}\right)$ is unknown, so a mathematical model should be established to calculate its value. Set $z=X^{2}$ and calculate the value of $D(Z)$.

In mathematical statistics, the relationship between the expectation $E(X)$ and the variance $D(X)$ is as follows:

$$
D(X)=E\left(X^{2}\right)-[E(X)]^{2}
$$

so

$$
D(Z)=D\left(X^{2}\right)=E\left(X^{4}\right)-\left[E\left(X^{2}\right)\right]^{2}
$$

In (8), only $E\left(X^{4}\right)$ is unknown and it can be calculated by (9):

$$
E\left(X^{4}\right)=\int_{-\infty}^{+\infty} X^{4} f(X) d X
$$


where $f(X)$ is given by (1). Therefore, (9) can be rewritten as:

$$
E\left(X^{4}\right)=\int_{-\infty}^{+\infty} X^{4} \frac{1}{\sqrt{2 \pi} \times 31.78} e^{-\frac{(X-48.48)^{2}}{2 \times 31.78^{2}}} d X
$$

Programming (10) in Matlab, we obtain $E\left(X^{4}\right)=2.2916 \times 10^{7} \quad$. Insert it and $E\left(X^{2}\right)=3360.3 \quad$ into (8), we have $D(Z)=1.16 \times 10^{7}$. Since we have obtained the values of $D(X)$ and $D\left(X^{2}\right)$ as well as $a, b$, according to (6), it is easy to work out $D(Y)=1.75 \times 10^{4}$.

To sum up, the expectation and variance of acceleration peak value are 76.86 and $1.75 \times 10^{4}$, respectively. Kinetic energy will be dispersed in a very short period of time when vehicle collision accidents occur. The loss in the process of vehicle collision can be minimized by a reasonable vehicle safety design. Therefore, it is very helpful to optimize the safety design of the vehicle based on the expectation and variance of peak value of acceleration.

\section{CONCLUSIONS}

In this paper a large number of collision accidents are analyzed. The results show that the vehicle collision speeds generally follow a normal distribution. At low and medium speeds, the probability of vehicle collision accident increases as the increase of vehicle speed. It is at $48.48 \mathrm{~km} / \mathrm{h}$ that the probability of vehicle collision accident reaches maximal value. Then, the collision probability decreases with an increasing speed. The values of acceleration peaks at multiple speeds are obtained using a finite element model of trolley impact system. Then, we obtain the regression model for acceleration peak in terms of the collision speed. Furthermore, based on data of the collision speeds and acceleration peaks, the numerical characteristics of acceleration peak, including expectation and variance, are calculated, which are useful for optimizing of automobile safety system under the condition of random impact.

There are several possibilities for furthering our research on this topic area. First, one could research the impact of features of vehicle on the acceleration peak. Note that in this paper we carry out our research using simulation model rather than real vehicle collision data. Generally speaking, the features of vehicles (such as body structure, body material and so on) are bound to affect the acceleration peak when a collision takes place. However, such analysis is technically complex since there are too many parameters need to be taken into consideration. Second, one could consider omitting the negative tail of normal distribution of collision speed. Note that in this paper, we do the integral in the interval from negative infinity to positive infinity, whereas it is impossible for collision speeds to be less than zero.

\section{ACKNOWLEDGMENT}

The authors wish to thank the referee for his/her suggestions that improved an earlier version of this paper. This work was supported in part by Natural Science Foundation of China (no. 51605197), in part by Natural Science Foundation of Jiangsu Province (no. BK20160524) and in part by China Postdoctoral Science Foundation (no. 2015M571691).

\section{REFERENCES}

[1] H. Fagerlind, J. Brown, and A. Bálint, "A novel approach to study the health consequences of road crashes," Journal of Transport \& Health, vol. 7, pp. 280-287, October 2017.

[2] H. Huang, C. Li, and Q. Zeng, "Crash protectiveness to occupant injury and vehicle damage: An investigation on major car brands," Accident Analysis \& Prevention, vol. 86, pp. 129-136, January 2016.

[3] Y. Zou and A. P. Tarko, "Barrier-relevant crash modification factors and average costs of crashes on arterial roads in Indiana," Accident Analysis \& Prevention, vol. 111, pp. 71-85, January 2018.

[4] R. Hamzeie, P. T. Savolainen, and T. J. Gates, "Driver speed selection and crash risk: Insights from the naturalistic driving study," Journal of Safety Research, vol. 63, pp. 187-194, November 2017.

[5] Q. Zeng, H. Wen, and H. Huang, "The interactive effect on injury severity of driver-vehicle units in two-vehicle crashes," Journal of Safety Research, vol. 59, pp. 105-111, October 2016.

[6] A. Bener, E. Yildirim, T. Özkan, and T. Lajunen, "Driver sleepiness, fatigue, careless behavior and risk of motor vehicle crash and injury: Population based case and control study," Journal of Traffic and Transportation Engineering (English Edition), vol. 4, pp. 496-502, September 2017.

[7] M.-I. M. Imprialou, M. Quddus, D. E. Pitfield, and D. Lord, "Re-visiting crash-speed relationships: A new perspective in crash modelling," Accident Analysis \& Prevention, vol. 86, pp. 173-185, January 2016.

[8] A. Vadeby and A. Forsman, "Changes in speed distribution: Applying aggregated safety effect models to individual vehicle speeds," Accident Analysis \& Prevention, vol. 103, pp. 20-28, March 2017.

[9] W. Hu, "Raising the speed limit from 75 to $80 \mathrm{mph}$ on Utah rural interstates: Effects on vehicle speeds and speed variance," Journal of Safety Research, vol. 61, pp. 83-92, March 2017.

[10] Z.-x. Li, J. Shen, Y. Lu, and W. Xiao, "Study on trigger algorithm of automatic crash notification system based on power Density," Science Technology and Engineering, vol. 15, pp. 84-88, September 2015 (in Chinese). 